\title{
Successful PerOral Endoscopic Myotomy (POEM) After Three Failed Heller in a 62-Years-Female with Sigmoid Type II Achalasia and Mega-esophagus
}

\author{
Nikolas Eleftheriadis ${ }^{1 *}$, Haruo Ikeda ${ }^{2}$, Manabu Onimaru ${ }^{2}$, Ioanna Bravita ${ }^{1}$, Damianos Eleftheriadis ${ }^{1}$ and Haruhiro \\ Inoue $^{2}$ \\ ${ }^{1}$ Kyanous Stauros Euromedica, Thessaloniki, Greece \\ ${ }^{2}$ Digestive Diseases Center, Showa University, Koto Toyosu Hospital, Japan \\ *Corresponding Author: Nikolas Eleftheriadis, Kyanous Stauros Euromedica, Thessaloniki, Greece.
}

Received: August 13, 2019; Published: September 23, 2019

DOI: 10.31080/ASGIS.2019.02.0080

\section{Abstract}

Aim: To report on the successful application of PerOral Endoscopic Myotomy (POEM) in a 62-years-old female, with complex sigmoid type II achalasia, mega-esophagus and failure of three previous surgical Heller myotomies.

Patient and Methods: A 62-years-old female with 15-years history of esophageal achalasia and three previous failed Heller myotomies, came for persistent severe dysphagia, total feeding failure, weight loss $>10 \mathrm{~kg}$ and frequent hospitalizations due to recurrent aspiration pneumonias and food impactions the last months. Eckard score was 12.

Esophagogram showed sigmoid type II achalasia, mega-esophagus (esophageal diameter $>6 \mathrm{~cm}$ ) and failure of passage of contrast medium to stomach. Gastroscopy showed large amount of solid food impacted in the dilated esophagus.

Due to severe deterioration of her symptoms, in combination with failure of all other previous treatments including surgical Heller myotomy, and in view of absence of other realistic acceptable therapeutic option, patient agreed to undergo the innovative minimally invasive POEM procedure.

Results: POEM was performed under general anesthesia with intubation, according to previous description. During POEM CO2 insufflation and TT-J knife was used. Despite the extraordinary technical difficulties of this particular case, due to sigmoid type II, with mega-esophagus and complications from previous surgeries, POEM was successfully completed in all stages. Finally, posterior circular myotomy $13 \mathrm{~cm}$ in length was successfully completed, with $2 \mathrm{~cm}$ extension to the gastric side below GEJ. All intraoperative complications (bleeding, pneumomediastinum) were successfully managed, while no other severe acute or late complications were reported. Patient started drinking next day and discharged two days after POEM, with gradual increase in diet from liquids to soft, semi-solid and solid diet, during following weeks after POEM. Her dysphagia was completely disappeared soon after POEM. During follow-up one year later, patient is in a good general condition, returned to normal life, with weight gain and complete disappearance of dysphagia.

Conclusion: We consider the POEM technique minimally invasive, feasible, safe, and effective therapy, for complex achalasia patients, such as sigmoid type II achalasia, mega-esophagus and after failure of previous Heller myotomy.

Keywords: Sigmoid Type II Achalasia; Failed Heller; POEM

\section{Introduction}

POEM has been considered the most modern, innovative, minimal invasive, long-term treatment for all types of esophageal achalasia [1]. However, little experience exist on the application of POEM for complex achalasia patients [2], particularly for sigmoid type II achalasia [2-4] or after failure of all other existing treatment options, such as after failed surgical or laparoscopic Heller (LHM) myotomy [2,4-6].

Sigmoid type II achalasia, mega-esophagus, as well as failure of previous surgical myotomy, makes further treatment options difficult, while there is no international consensus for the therapy of choice in these complex and severe achalasia cases. Esophagectomy could be proposed as the ultimate solution, however with high morbidity and mortality rate [7].

The aim of the present study is to report on the successful application of POEM in a 62-years-old female with complex sigmoid type II achalasia, mega-esophagus and failure of three previous surgical Heller myotomies.

\section{Patient and Methods}

We report the safe and successful application of the POEM procedure in a 62-years-old female with 15-years history of esophageal achalasia and three previous failed Heller myotomies, who 
came for persistent severe dysphagia, total feeding failure, weight loss $>10 \mathrm{~kg}$ and frequent hospitalizations due to recurrent aspiration pneumonias and food impactions the last months.

She reported initial surgical Heller myotomy in 2005, with slight improvement of dysphagia for 10 years. One year ago patient underwent a second laparoscopic Heller myotomy (LHM), due to severe \deterioration of dysphagia to liquid as well as solid foods, without however improvement. Some months after 2nd LHM she underwent a 3rd surgical Heller myotomy in combination with antireflux Toupet fundoplication and gastropexy. The 3rd surgical procedure was complicated by anastomotic leak and bilateral pleural infusion, which was managed by esophageal stent placement, prolonged hospitalization, long-term fasting, intravenous antibiotics and long-term total parenteral nutrition (TPN).

Despite all these previous surgeries, dysphagia persisted and deteriorated, and patient continued to suffer from total feeding failure, severe weight loss $>10 \mathrm{~kg}$. She also reported frequent, weekly, hospitalizations for recurrent endoscopic removal of impacted solid foods in the dilated esophagus and intravenous nutrition, the last months. Her general condition was also deteriorated. Eckard score was 12 (stage III).

Esophagogram showed sigmoid type II achalasia with megaesophagus (esophageal diameter $>6 \mathrm{~cm}$ ) and failure of passage of contrast medium to stomach (Figure 1C). Gastroscopy showed large amount of solid foods impacted in the dilated esophagus, (Figure 1A, B), while endoscopic clearance of the esophagus from solid foods was demanding and time-consuming. Sutures from gastropexy were identified at the gastroesophageal junction (GEJ), which aggravated esophageal motility, while respiration resulted in increase esophageal movements and severe food regurgitation from the dilated esophagus (Figure 1 A, B).

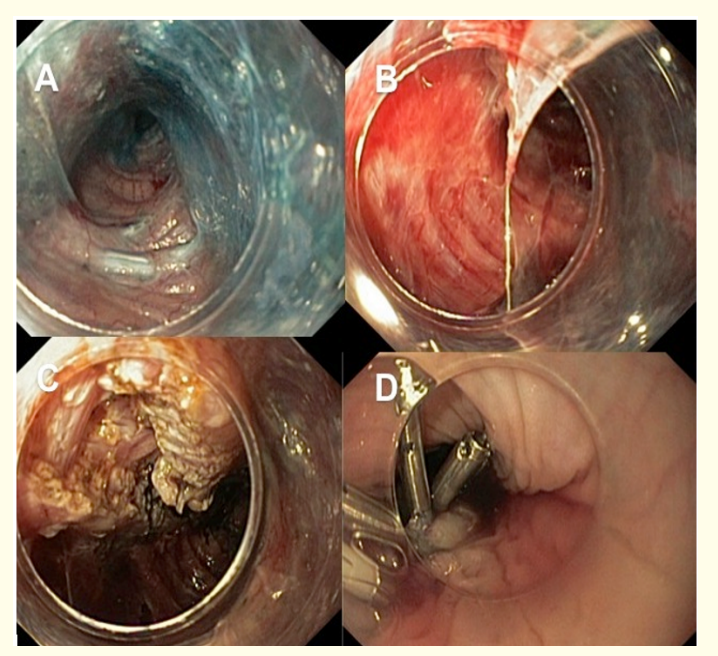

Figure 1: A, B) Submucosal tunnel. C) Myotomy.

D). Closure of mucosal entry by clips.
Due to severe deterioration of her symptoms, in combination with debilitating general condition, failure of all other previous treatments including surgical Heller myotomies and in view of absence of other realistic acceptable therapeutic options, patient agreed to undergo the innovative minimally invasive POEM procedure.

\section{Results}

POEM was performed under general anesthesia with intubation, according to previous description1. During POEM, CO2 insufflation and TT-J knife was used (Figure 2A-D).

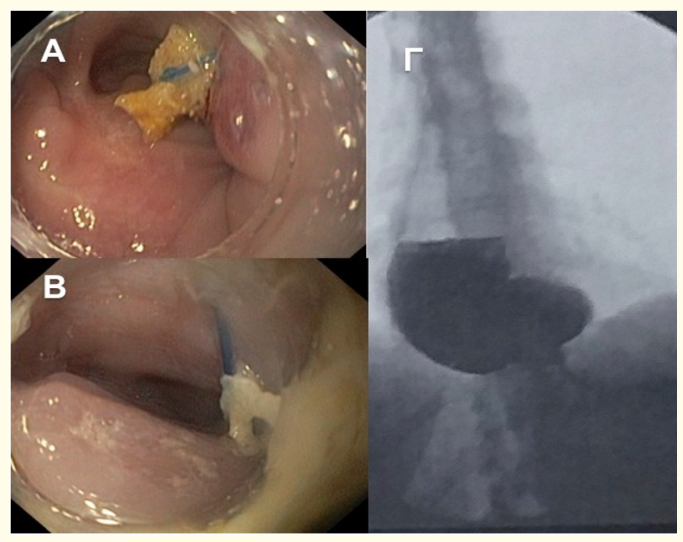

Figure 2: A, B) Dilated esophagus, food stasis in the esophagus and sutures from previous Heller and gastropexy. C) Sigmoid type II mega-esophagus

Despite the extraordinary technical difficulties of this particular case, due to sigmoid type II, mega-esophagus and complications from previous surgeries, POEM was successfully completed in all stages. Particularly, submucosal tunneling and muotomy were extremely difficult and time-consuming, due to sigmoid megaesophagus in combination with the immobilization of the lower esophagus and respiratory esophageal movements due to previous gastropexy. Total POEM time was $125 \mathrm{~min}$. Sutures from previous gastropexy were also identified in the subumucosal tunnel. Moreover, due to tension at the lower esophagus intense bleeding in the tunnel was observed with creation of large clot in the tunnel, which was removed and then hemostasis was succeeded using coag-grasper, with no further consequences.

Finally, posterior circular myotomy $13 \mathrm{~cm}$ in length was successfully completed (Figure 2C), from $30-43 \mathrm{~cm}$ from dentate line, with $2 \mathrm{~cm}$ extension to the gastric side below the GEJ. Circular muscle layer was extremely thick (Figure 2C), while mucosal entry closure at the end of POEM was also extremely difficult due to tension from sigmoid and dilated esophagus. Finally closure was succeeded, using 7 large diameter clips (16m).

All intraoperative complications (bleeding) (Figure 3A) were successfully managed, while no other severe acute or late complica-

Citation: Nikolas Eleftheriadis., et al. "Successful PerOral Endoscopic Myotomy (POEM) After Three Failed Heller in a 62-Years-Female with Sigmoid Type II Achalasia and Mega-esophagus". Acta Scientific Gastrointestinal Disorders 2.8 (2019): 14-17. 
tions were reported. Esophagogram after POEM showed rapid passage of contrast medium to the stomach (Figure 3B). Patient started drinking next day and discharged two days after POEM, with gradual increase in diet from liquids to soft, semi-solid and solid diet, during following weeks after POEM. Her dysphagia was completely disappeared soon after POEM. During follow-up one year later, patient is in a good general condition, returned to normal life, with weight gain and complete disappearance of dysphagia (figure 3 C-D), while no reflux symptoms or endoscopic findings of reflux esophagitis were found and took no medications.

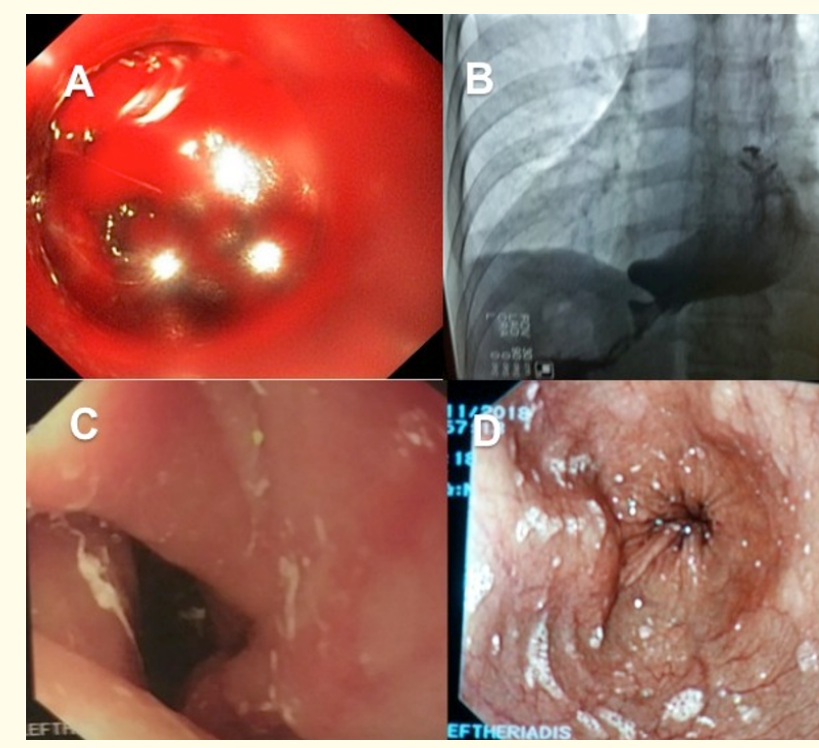

Figure 3: A) Bleeding inside tunnel. B) Rapid passage of contrast medium after POEM. C, D). GEJ remains open one year after POEM.

\section{Discussion and Conclusion}

The results of the present report were comparable to international standards $[2,8]$, in terms of feasibility, safety and efficacy of POEM technique. POEM performed during endoscopy, is a minimally invasive procedure, that can be applied even to debilitating patients, with severe weight loss, poor general condition and high risk for surgery, as in our case. Another advantage is the short hospitalization time ( 2 days in our case) and rapid recovery time (patient mobilized the next day and returned to her daily activities within a week).

Other major advantages of POEM, as in our case, are the posterior access and the longest myotomy length $(13 \mathrm{~cm}$ in our case, from $30-43 \mathrm{~cm}$ from dentate line), which are practically impossible with any other method. Posterior POEM has the advantage to approximate the diseased circular esophageal muscle, directly and easily through the submucosal tunnel, permitting safe and efficient long myotomy, avoiding at the same time the scar from previous anterior surgical myotomy and sutures from previous gastropexy.
It is worthy to notice that previous surgical Heller myotomies, not only made POEM techically more difficult, but increase the risk of severe post POEM complications.

Another important advantage of POEM, is that no anti-reflux procedure is absolutely necessary after POEM, because the normal antireflux mechanisms (diafragmatic crue and angle of His) remain intact during POEM due to internal access through the submucosal tunnel. This is of great importance particularly in complex achalasia cases, such as in our case, because additional antireflux procedure after POEM, could increase operation time and risk of complications, while makes POEM more invasive.

In conclusion, according to our rare case, in combination with scarce international experience and abscence of consensus regarding therapy of choice in similar cases, we consider POEM safe, effective and feasible realistic treatment even for complex achalasia patients (sigmoid type II, mega-esophagus, failled Heller myotomies). In such severe achalasia cases, POEM is not an alternative to other existing therapies, but to our opinion, is the only realistic therapeutic option, provided long-term solution. However, further international experience and long-term follow-up is necessary and awaited.

\section{Bibliography}

1. Inoue H., et al. "Peroral endoscopic myotomy (POEM) for esophageal achalasia”. Endoscopy 42 (2010): 265-271.

2. Bechara R., et al. "Peroral endoscopic myotomy (POEM) for complex achalasia and the POEM difficulty score". Digestive Endoscopy 31 (2019): 148-155.

3. Onimaru M., et al. "Greater curvature myotomy is a safe and effective modified technique in per-oral endoscopic myotomy (with videos)". Gastrointestinal Endoscopy (2015).

4. Eleftheriadis N and Eleftheriadou ED. "Successful peroral endoscopic myotomy performed in Endoscopy Department as a radical, long-term treatment for esophageal achalasia - the Greek experience". Therapeutics and Clinical Risk Management 13 (2017): 185-190.

5. Onimaru M., et al. "Peroral endoscopic myotomy is a viable option for failed surgical esophagocardiomyotomy instead of redo surgical Heller myotomy: a single center prospective study". Journal of the American College of Surgeons 217 (2013): 598-605.

6. Zhou PH., et al. "Peroral endoscopic remyotomy for failed Heller myotomy: a prospective single-center study". Endoscopy 45 (2013): 161-166 
7. Luketich JD., et al. "Outcomes after minimally invasive esophagectomy: review of over 1000 patients". Annuals of Surgery 256 (2012): 95-103.

8. Stavropoulos SN., et al. "The International Per Oral Endoscopic Myotomy Survey (IPOEMS): a snapshot of the global POEM experience". Surgical Endoscopy 27 (2013): 3322-3338.

\section{Volume 2 Issue 8 October 2019}

(C) All rights are reserved by Nikolas Eleftheriadis.,

et al. 